\title{
Mechanisms for the induction of gastric cancer by Helicobacter pylori infection: aberrant DNA methylation pathway
}

\author{
Masahiro Maeda $^{1,2} \cdot$ Hiroshi Moro ${ }^{1,3} \cdot$ Toshikazu Ushijima ${ }^{1}$
}

Received: 12 September 2016/Accepted: 23 September 2016/Published online: 7 October 2016

(c) The International Gastric Cancer Association and The Japanese Gastric Cancer Association 2016

\begin{abstract}
Multiple pathogenic mechanisms by which Helicobacter pylori infection induces gastric cancer have been established in the last two decades. In particular, aberrant DNA methylation is induced in multiple driver genes, which inactivates them. Methylation profiles in gastric cancer are associated with specific subtypes, such as microsatellite instability. Recent comprehensive and integrated analyses showed that many cancer-related pathways are more frequently altered by aberrant DNA methylation than by mutations. Aberrant DNA methylation can even be present in noncancerous gastric mucosae, producing an "epigenetic field for cancerization." Mechanistically, $H$. pylori-induced chronic inflammation, but not $H$. pylori itself, plays a direct role in the induction of aberrant DNA methylation. The expression of three inflammation-related genes, Illb, Nos2, and Tnf, is highly associated with the induction of aberrant DNA methylation. Importantly, the degree of accumulated aberrant DNA methylation is strongly correlated with gastric cancer risk. A recent multicenter prospective cohort study demonstrated the utility of epigenetic cancer risk diagnosis for metachronous gastric cancer. Suppression of aberrant DNA methylation by a demethylating agent was shown to inhibit gastric
\end{abstract}

Toshikazu Ushijima

tushijim@ncc.go.jp

Masahiro Maeda

mamaeda@ncc.go.jp

1 Division of Epigenomics, National Cancer Center Research Institute, Tokyo, Japan

2 Department of Gastrointestinal Surgery, Kyoto University Graduate School of Medicine, Kyoto, Japan

3 Department of Gastric Surgery, National Cancer Center Hospital, Tokyo, Japan cancer development in an animal model. Induction of aberrant DNA methylation is the major pathway by which $H$. pylori infection induces gastric cancer, and this can be utilized for translational opportunities.

Keywords Helicobacter pylori infection - Aberrant DNA methylation $\cdot$ Epigenetic cancer risk diagnosis

\section{Introduction}

'How does Helicobacter pylori infection induce gastric cancer?' has long been a challenging question. For the last two decades, various pathogenic mechanisms of $H$. pyloriassociated gastric cancer have been intensively investigated, and three major mechanisms have become clear. First, multiple signaling pathways were shown to be perturbed in gastric epithelial cells by virulence factors of $H$. pylori such as VacA and CagA $[1,2]$. This mechanism is closely involved in the $H$. pylori type IV secretion machinery. Second, mutations were shown to be induced by aberrant expression of activation-induced cytidine deaminase (AID) via NFKB activation in gastric epithelial cells due to $H$. pylori infection-induced chronic inflammation [3]. Third, aberrant DNA methylation was shown to be accumulated in gastric mucosa by chronic inflammation caused by $H$. pylori infection [4].

In particular, multiple lines of evidence indicate that the accumulation of aberrant DNA methylation is very important in gastric carcinogenesis. Firstly, aberrant DNA methylation, a representative epigenetic alteration, can cause inactivation of tumor-suppressor genes. Indeed, comprehensive and integrated analyses of gastric cancer have shown that aberrant DNA methylation has a major impact $[5,6]$. Secondly, the degree of accumulation of 
aberrant DNA methylation is highly correlated with gastric cancer risk [7, 8]. Furthermore, animal experiments have shown that inhibiting aberrant DNA methylation induction could prevent gastric cancer development [9].

In this review, we provide an overview of the current understanding of the mechanisms by which aberrant DNA methylation is induced by $H$. pylori infection. We also highlight potential applications of aberrant DNA methylation in precision medicine.

\section{Deep involvement of aberrant DNA methylation in gastric cancer}

Genetic and epigenetic alterations accumulate during multistep carcinogenesis through exposure to various carcinogenic factors [10]. However, few frequent driver mutations associated with gastric cancer have been identified besides TP53 and CDH1. Although several new driver genes such as ARIDIA and RHOA have been identified by recent exome and whole-genome sequencing $[5,11]$, such mutations account for less than $15 \%$ of all gastric cancers. Indeed, more than $20 \%$ of gastric cancers present only one or even no mutation [11].

On the other hand, a deep involvement of aberrant DNA methylation in gastric cancer has been highlighted [12]. In 1999, frequent occurrence of aberrant DNA methylation of $\mathrm{CpG}$ islands ( $\mathrm{CpG}$ island methylator phenotype; CIMP) was shown to be associated with microsatellite instability in gastric cancer, as it is in colon cancer [13]. Characteristically, Epstein-Barr virus-positive gastric cancer has been known to display extreme CIMP [14, 15]. These findings were validated by The Cancer Genome Atlas (TCGA) Research Network [5]. Furthermore, a recent integrated analysis of genetic and epigenetic alterations revealed that inactivation of tumor-suppressor genes such as $p 16, h M L H 1$, and $\mathrm{CDH} 1$ and activation of the WNT pathway were more frequently caused by aberrant DNA methylation than by mutations (Fig. 1) [6]. This evidence suggests that aberrant DNA methylation has as much or even more of an impact on gastric carcinogenesis than mutations.

\section{DNA methylation level in noncancerous mucosa and gastric cancer risk}

Aberrant DNA methylation can even be present in noncancerous gastric mucosa, and its levels are influenced by H. pylori infection (Fig. 2). The association of aberrant DNA methylation in gastric mucosa with $H$. pylori was reported by Chan et al. for the first time in 2003 [16]. However, at the same time, Kang et al. showed that there was no association between them [17]. These conflicting findings were considered to be due to nonquantitative DNA methylation analyses. Later, a quantitative methylation analysis focusing on $\mathrm{CpG}$ islands of passenger genes clearly demonstrated an association between high methylation levels in gastric mucosae and $H$. pylori infection [7].

At the same time, a cross-sectional study suggested that eradicating $H$. pylori leads to a decrease in DNA methylation levels [7]. Later, temporal analyses showed that eradication of $H$. pylori decreases DNA methylation levels [18-21]. Importantly, among individuals not currently infected with $H$. pylori, DNA methylation levels were much higher in cancer patients than in healthy individuals [7]. Additionally, methylation levels were higher in cases with multiple gastric cancers than in cases with a single cancer [8]. It was therefore suggested that DNA methylation levels in individuals not currently infected with $H$. pylori are closely correlated with gastric cancer risk.

\section{Cell types and genes susceptible to aberrant DNA methylation}

Gastric mucosal biopsy samples contain various types of cells in addition to epithelial cells. Therefore, the cell types that aberrant DNA methylation is induced in were unclear. This issue was addressed by observing increased methylation levels of multiple genes in gastric epithelial cells highly purified by the gland isolation technique from the stomachs of Mongolian gerbils (Meriones unguiculatus), a widely used animal model for $H$. pylori infection and gastric cancer [4]. Also, in a genome-wide DNA methylation analysis of human gastric mucosa, aberrant DNA methylation was still observed, even after the exclusion of CpG sites methylated in human blood cells, ruling out the possibility of increased methylation due to blood cellspecific methylation (Nanjo et al., unpublished data). These data showed that gastric epithelial cells are the real targets of aberrant DNA methylation induction. Nevertheless, there remains the possibility that aberrant DNA methylation is also induced in other types of cells, such as stromal cells, and that such epigenetic alterations may also be important for gastric cancer development.

Eradicating $H$. pylori decreases DNA methylation levels in gastric mucosae, and the decreased methylation levels persist for a long time [4, 20]. This suggests that aberrant DNA methylation consists of transient and permanent components. Mechanistically, we can speculate that the aberrant DNA methylation induced in stem cells of a gastric gland is a permanent component because methylation status in stem cells is preserved and replicated, thus determining the fraction of cells with methylation. In contrast, methylation induced only in differentiated cells will disappear when they are replaced by new cells without 
Fig. 1 Genetic and epigenetic alterations of genes in multiple signaling pathways in 6 normal gastric mucosa and 50 gastric cancer samples. Three growthpromoting and four tumorincluded. Inactivation of tumorsuppressor genes such as $p 16$, $h M L H 1$, and $C D H 1$ and activation of the WNT pathway were more frequently caused by aberrant DNA methylation than by mutations. This figure was modified from [6] suppressor pathways are

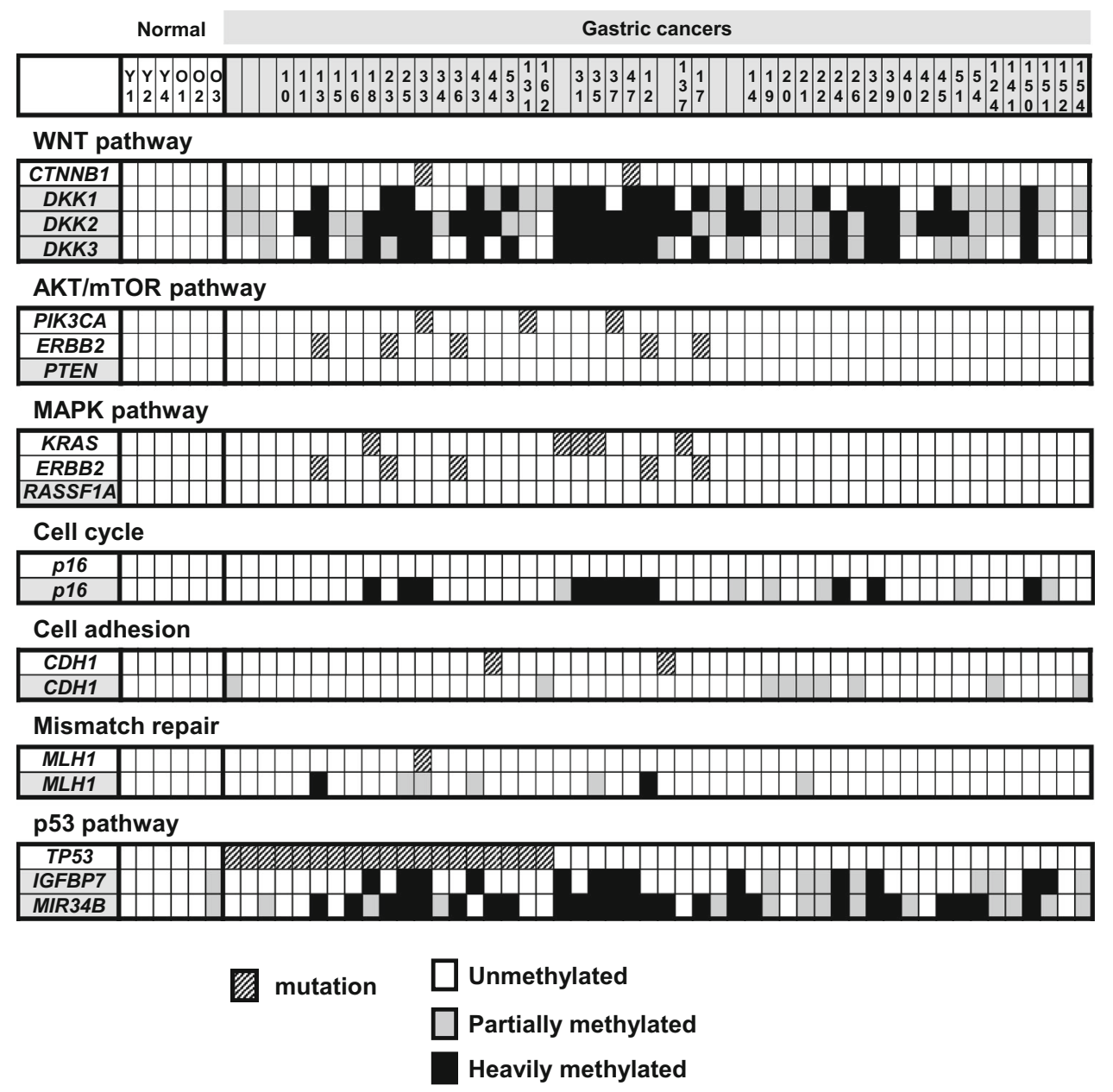

DNA methylation induction, and gastric cancer risk. The clinical course of individuals infected with $H$. pylori is illustrated. The H. pylori infection occurs in childhood, causing chronic inflammation in the stomach. Chronic inflammation induces aberrant DNA methylation in gastric mucosa. Once H. pylori is eradicated, the DNA methylation level decreases somewhat but does not disappear completely. The degree of residual DNA methylation is strongly correlated with gastric cancer risk

\section{H. pylori infection status}

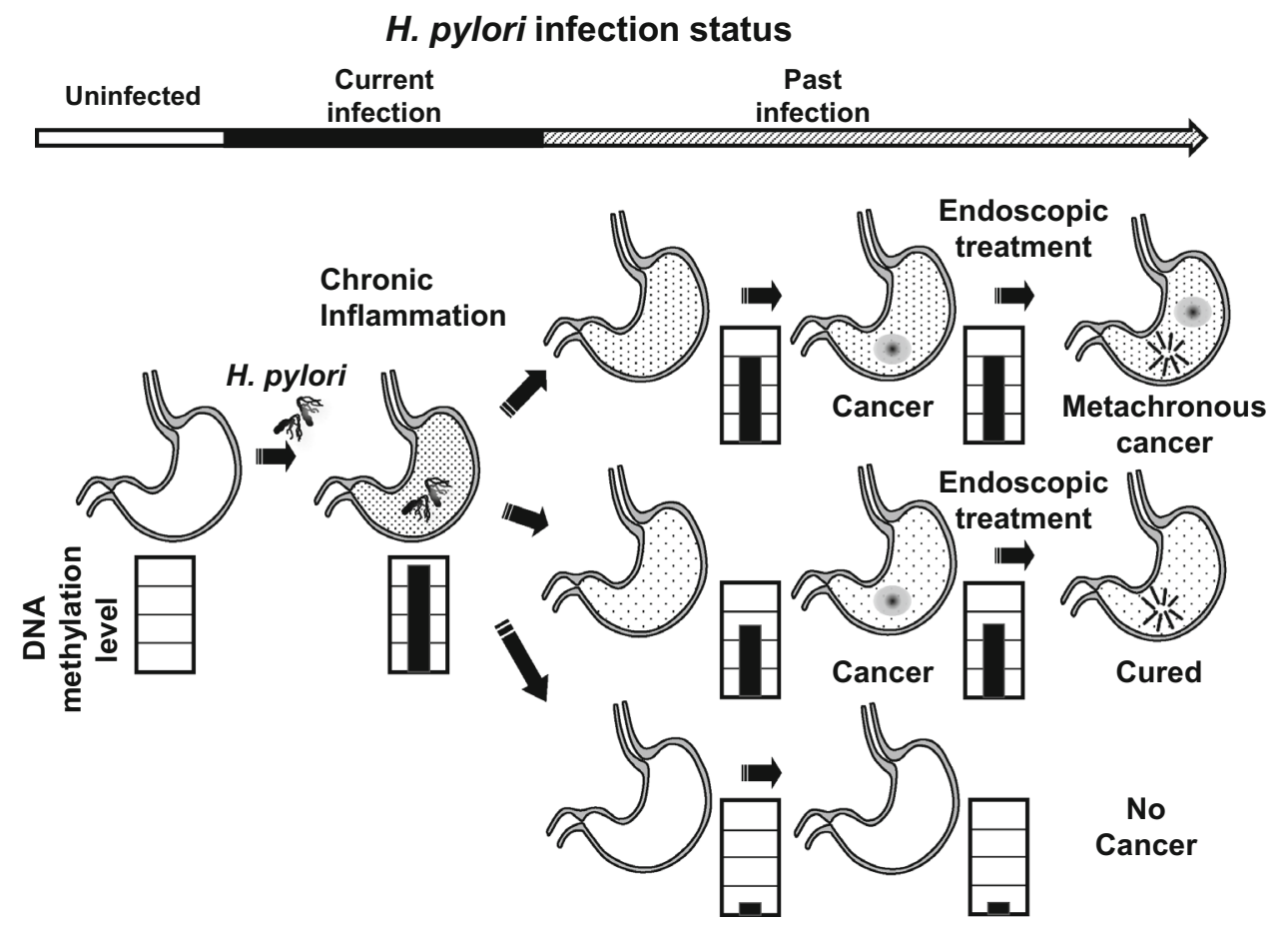


methylation derived from a stem cell without methylation, meaning that this methylation induced in differentiated cells represents a transient component [22] (Fig. 3).

A large number of specific genes are aberrantly methylated by $H$. pylori infection [23]. Mechanistically, it is generally known that promoter $\mathrm{CpG}$ islands without active transcription of their downstream genes and with a specific histone modification, $\mathrm{H} 3 \mathrm{~K} 27 \mathrm{me} 3$, are likely to become methylated [24-26]. In addition to physiological H3K27me3, aberrant H3K27me3 can be induced by environmental factors such as chronic inflammation [27]. Therefore, in gastric mucosa, genes that are not expressed naturally or those that are downregulated by $H$. pylori infection are likely to become methylated.

Such genes that are not expressed naturally are considered to play no biological role in gastric mucosae. Therefore, the methylation of such genes is likely to have no biological consequences in gastric carcinogenesis, and is thus considered a passenger event. On the other hand, although driver genes such as $p 16, C D H 1$, and $M L H 1$ are expressed in gastric mucosae with diverse expression levels, they are methylated in cancer cells. If we identify genes that are methylated in gastric cancer but expressed in normal gastric mucosae, they are more likely to be driver genes [28].

\section{Mechanisms by which $H$. pylori infection induces aberrant DNA methylation}

To verify that $H$. pylori infection induces aberrant DNA methylation, Mongolian gerbils were infected with $H$. pylori, and induction of aberrant DNA methylation in

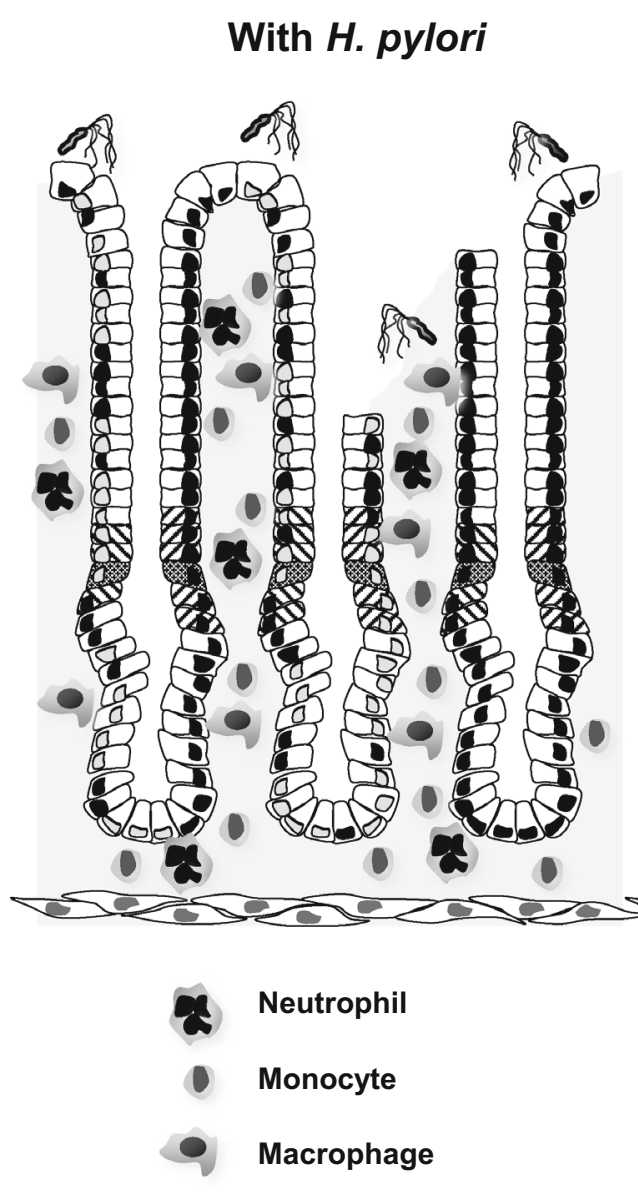

Fig. 3 Potential target cells for the induction of aberrant DNA methylation. Left: gastric mucosa with active $H$. pylori infection. Right: gastric mucosa after eradication of $H$. pylori. Chronic inflammation, characterized by infiltration of monocytes/macrophages with neutrophils, induces aberrant DNA methylation. Aberrant DNA methylation is actively induced in differentiated cells, possibly in progenitor cells (transient component), along with some stem cells. When methylation is present in a stem cell, all of the cells derived from the stem cell in a gland are

\section{After eradication}
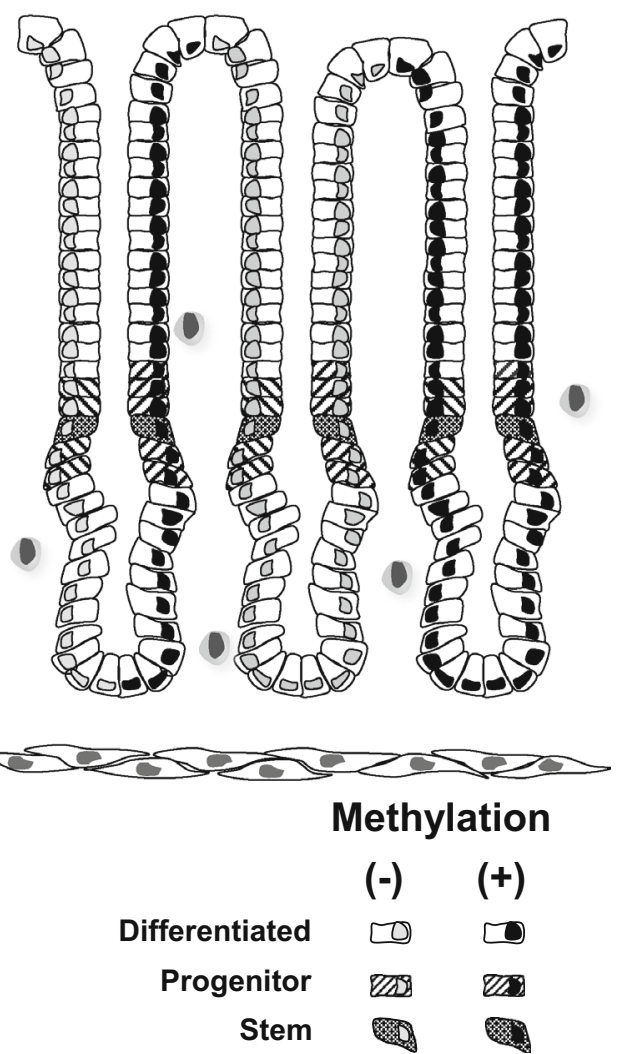

methylated (permanent component). When methylation is induced in differentiated cells, heterogeneous methylation within a gland is present, and this methylation will disappear when fresh cells without methylation are derived from a stem cell. Without active induction of aberrant DNA methylation, the methylation status of a gland reflects that of its stem cell. The methylation level in the gastric mucosa is assumed to be proportional to the fraction of stem cells with methylation. This figure was modified from [22] 
purified gastric gland cells was demonstrated [4]. In addition, eradicating $H$. pylori clearly decreased methylation levels, which were accompanied by diminished histological inflammatory responses (Fig. 4a). Then, to address whether $H$. pylori or the resultant chronic inflammation was responsible for inducing aberrant DNA methylation, inflammatory responses were repressed by cyclosporin A, an immunosuppressive agent, in $\mathrm{H}$. pylori-infected gerbils. Although the amount of $H$. pylori was not reduced in the gastric mucosa, the repression completely suppressed the induction of aberrant DNA methylation [9]. Hence, it was concluded that it was not $H$. pylori itself but the inflammatory response triggered by $H$. pylori infection that was directly responsible for the induction of aberrant DNA methylation.
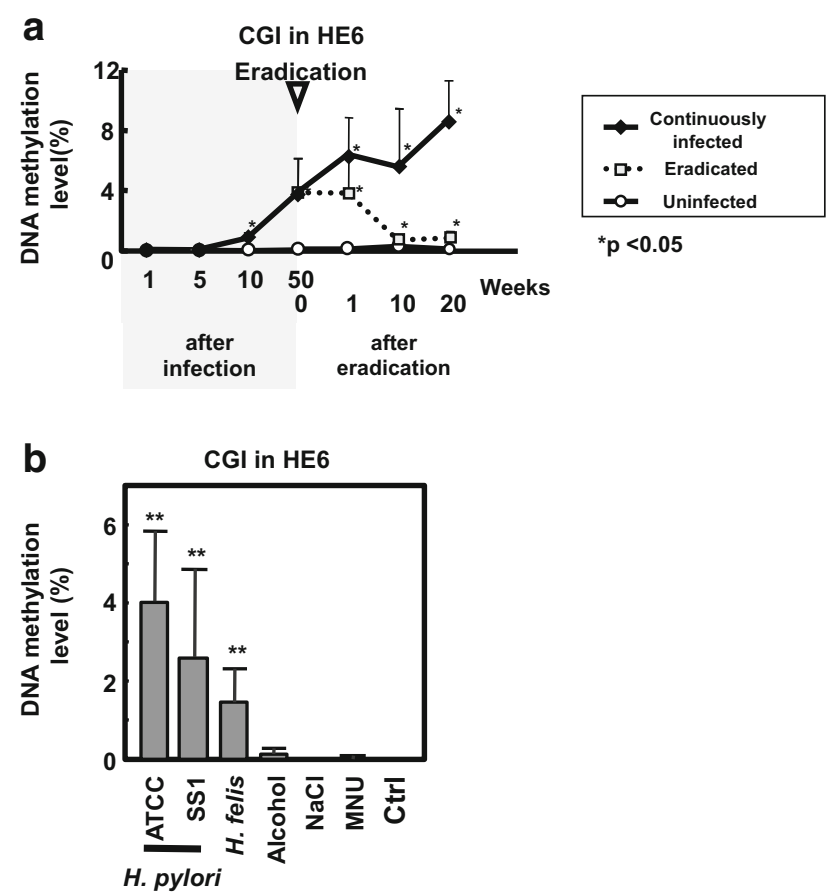

Fig. 4a, b Induction of aberrant DNA methylation by $H$. pylori infection in Mongolian gerbils and the effect of eradication. a After gerbils were infected with $H$. pylori, DNA methylation levels in purified gastric epithelial cells, as measured by quantitative methylation-specific PCR (qMSP), increased at $\geq 10$ weeks of infection. After eradication, DNA methylation levels were not decreased at 1 week, but were decreased at 10 and 20 weeks. Importantly, DNA methylation levels after eradication were still higher than those in never-infected gerbils. b Capacities of various kinds of inflammation to induce aberrant DNA methylation. Persistent inflammation was induced by $H$. pylori, H. pylori strain SS1, H. felis infection, high concentrations of alcohol, and saturated $\mathrm{NaCl}$. As controls, an MNU group and a nontreatment group were analyzed. In all eight $\mathrm{CpG}$ islands analyzed (methylation levels of $\mathrm{CpG}$ island HE6 are shown in Fig. 3b), only groups with H. pylori, H. pylori strain SS1, and H. felis infection showed the induction of aberrant DNA methylation. This figure was modified from [4]
The next question was whether any kind of persistent inflammation could induce aberrant DNA methylation. Mongolian gerbils were treated with alcohol or sodium chloride $(\mathrm{NaCl})$, both of which are well known to be inflammation inducers. Aberrant DNA methylation was induced only by $H$. pylori and $H$. felis, but not by high concentrations of alcohol or saturated $\mathrm{NaCl}$ (Fig. 4b). $H$. pylori and $\mathrm{H}$. felis triggered chronic inflammation as characterized by infiltration of monocytes/macrophages with residual neutrophils, whereas alcohol and $\mathrm{NaCl}$ elicited repeated acute inflammation as characterized by major infiltration of neutrophils [29].

Regarding inflammatory response genes, $I l 1 \mathrm{~b}, \mathrm{Nos} 2$, and $\operatorname{Tnf}$ were upregulated consistently in gastric mucosa of $H$. pylori- or $H$. felis-infected gerbils, and were associated with increased DNA methylation levels. Notably, $I l 1 b$ and Nos 2 were also induced in mouse colonic mucosae with dextran sulfate sodium-induced colitis [30]. Consequently, we can conclude that aberrant DNA methylation is induced by specific types of inflammation, and is likely to be associated with the expression of $I l 1 b, N o s 2$, and Tnf.

\section{Application of aberrant DNA methylation induced by $H$. pylori infection}

Aberrant DNA methylation in specific genes is frequent, even in noncancerous tissue, and contributes to carcinogenesis, so it could be used in a variety of applications relating to cancer risk diagnosis and chemoprevention.

\section{Clinical study of epigenetic cancer risk diagnosis}

The accumulation of aberrant DNA methylation in noncancerous tissues has been termed an "epigenetic field for cancerization" or "epigenetic field defect," especially in inflammation-associated cancers such as gastric cancer [31]. Cross-sectional studies have shown that the degree of a field defect can be assessed using appropriate cancer risk markers, as described above [32, 33]. However, crosssectional studies inevitably include various types of biases. Recently, a multicenter prospective cohort study for predicting the risk of metachronous gastric cancer demonstrated the utility of an epigenetic cancer risk marker for the first time [34].

In this study, gastric cancer patients were enrolled after endoscopic submucosal dissection (ESD). After assessing the methylation levels of three preselected genes, annual follow-up to detect metachronous gastric cancer was conducted for 3 years by trained endoscopists who were blinded to methylation information. Multivariate analysis showed that the highest quartile of the methylation level of $m i R-124 a-3$, a marker gene, had a significantly higher HR 


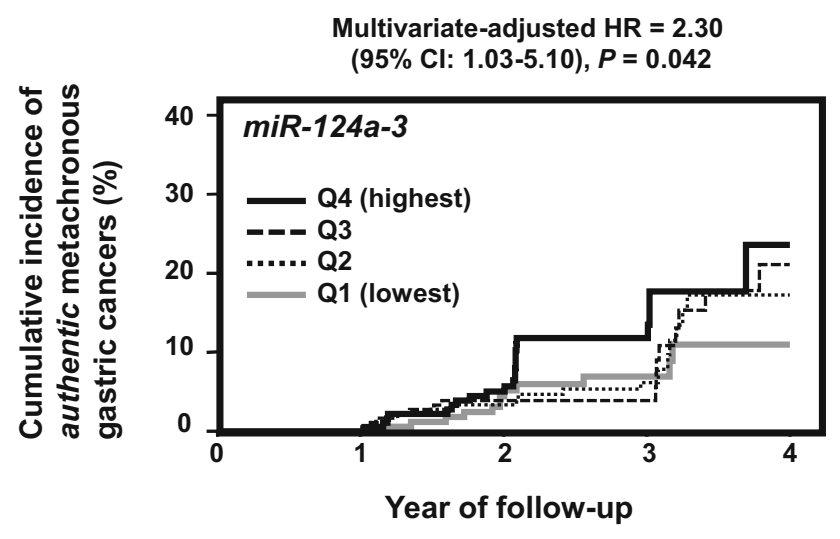

Fig. 5 Cumulative incidence of authentic metachronous gastric cancer (i.e., gastric cancer that developed after 1 year of enrollment). Patients were grouped into quartiles (Q1-Q4) based on methylation levels of $m i R-124 a-3$. Q4 (the highest) had a higher incidence of authentic metachronous gastric cancer than Q1 (the lowest). A multivariate analysis adjusting for hospital, gender, age, H. pylori infection before enrollment, pepsinogen index, past history of ER, smoking, and green vegetable intake showed that Q4 miR-124a-3 methylation had a higher HR than Q1 methylation $(95 \% \mathrm{CI})(2.30$ $(1.03-5.10) ; p=0.042)$. This figure was modified from [35]

of developing metachronous gastric cancer (Fig. 5) [35], showing that methylation levels can identify groups of patients at high risk for gastric cancer (Fig. 2).

That study achieved the proof-of-concept for epigenetic cancer risk diagnosis, but is unlikely to change clinical practice in relation to following up gastric cancer patients after ESD. In order to optimize a surveillance system based on individual risk, a new large-scale multicenter prospective cohort study (UMIN000016894) for predicting the risk of primary gastric cancer in healthy individuals after $H$. pylori eradication was proposed and is currently underway. The number of such healthy individuals is rapidly increasing in Japan after $H$. pylori eradication therapy was approved for chronic gastritis by the national health insurance [36].

\section{Application to cancer prevention}

Epigenetic alterations can be reversed by drug interventions and are therefore potential targets for chemoprevention. Importantly, a possible preventive effect of a DNA demethylating agent has been shown experimentally. Oral administration of a DNA demethylating agent, 5-aza-2'deoxycytidine (5-aza-dC), to $H$. pylori-infected gerbils treated with $N$-methyl- $N$-nitrosourea (MNU) reduced the incidence of gastric cancers from 55.2 to $23.3 \%$ (Fig. 6a), which was accompanied by a decrease in methylation levels (Fig. 6b) [9]. However, currently available DNA

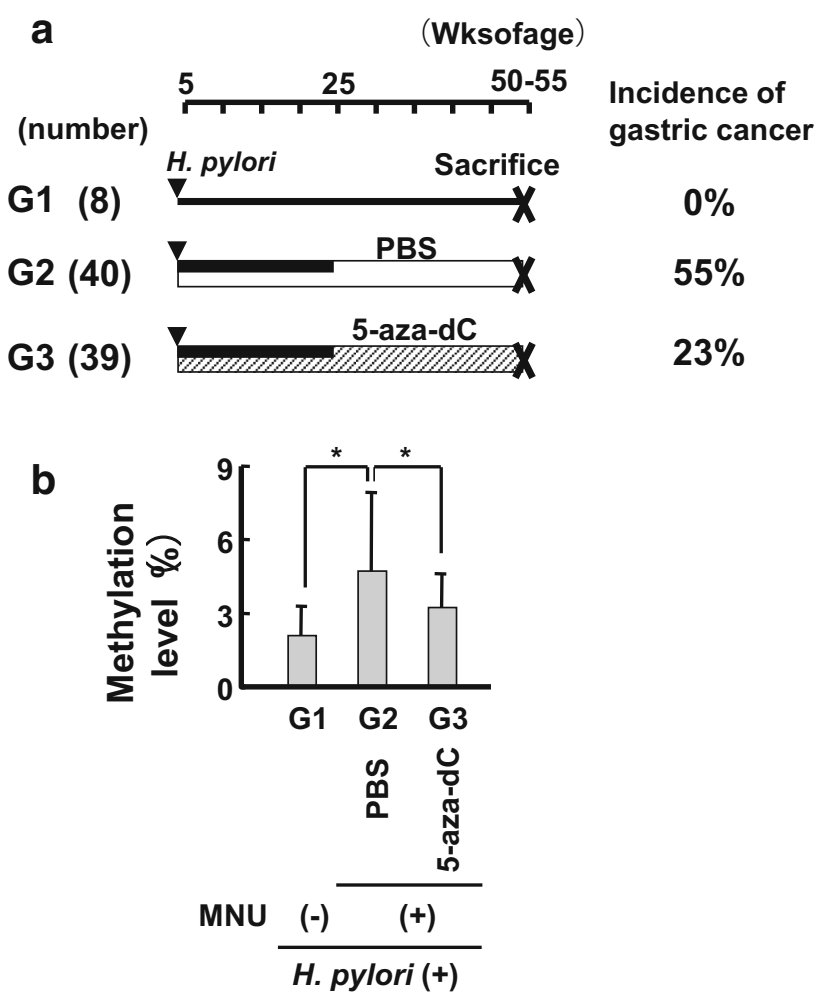

Fig. 6a, b Suppression of DNA methylation and inhibition of gastric cancers by 5 -aza-dC treatment in $H$. pylori-infected gerbils treated with MNU. a Protocol of the carcinogenicity experiment. The incidence of gastric cancer was reduced from $55 \%$ in group 2 of the H. pylori-infected gerbils with MNU to $23 \%$ in group 3, which received 5 -aza-dC $(p<0.05)$. b DNA methylation levels of $\mathrm{CpG}$ island HE6 in gastric epithelial cells (average \pm SD). DNA methylation levels were significantly lower in G3 than in $\mathrm{G} 2$. * $p<0.05$. This figure was modified from [9]

demethylating agents are not suitable for use in chemoprevention due to their adverse effects. Therefore, novel DNA demethylating agents with only minor adverse effects need to be developed, or intervention in an extremely highrisk population may be considered.

\section{Conclusions}

In this review, we have discussed the major impact of aberrant DNA methylation on gastric cancer and carcinogenesis, and current knowledge of the mechanisms for inducing aberrant DNA methylation. From the perspective of applying this knowledge, epigenetic cancer risk diagnosis is becoming a reality in the clinical setting. Clarification of the molecular mechanisms involved in aberrant DNA methylation induction is expected to provide a new strategy for the chemoprevention of gastric cancer. 


\section{Compliance with ethical standards}

Conflict of interest The authors declare that they have no conflict of interest.

Ethical standards This article does not contain any studies with human or animal subjects performed by any of the authors.

\section{References}

1. Amieva M, Peek RM Jr. Pathobiology of Helicobacter pyloriinduced gastric cancer. Gastroenterology. 2016;150(1):64-78. doi:10.1053/j.gastro.2015.09.004 (Epub 2015/09/20).

2. Hatakeyama M. Helicobacter pylori and gastric carcinogenesis. J Gastroenterol. 2009;44(4):239-48. doi:10.1007/s00535-0090014-1 (Epub 2009/03/10).

3. Matsumoto Y, Marusawa H, Kinoshita K, Endo Y, Kou T, Morisawa T, et al. Helicobacter pylori infection triggers aberrant expression of activation-induced cytidine deaminase in gastric epithelium. Nat Med. 2007;13(4):470-6. doi:10.1038/nm1566 (Epub 2007/04/03).

4. Niwa T, Tsukamoto T, Toyoda T, Mori A, Tanaka H, Maekita T, et al. Inflammatory processes triggered by Helicobacter pylori infection cause aberrant DNA methylation in gastric epithelial cells. Cancer Res. 2010;70(4):1430-40. doi:10.1158/0008-5472. can-09-2755 (Epub 2010/02/04).

5. The Cancer Genome Atlas Research Network. Comprehensive molecular characterization of gastric adenocarcinoma. Nature. 2014;513(7517):202-9. doi:10.1038/nature13480. http://www. nature.com/nature/journal/v513/n7517/abs/nature13480.html\#sup plementary-information

6. Yoda Y, Takeshima H, Niwa T, Kim JG, Ando T, Kushima R, et al. Integrated analysis of cancer-related pathways affected by genetic and epigenetic alterations in gastric cancer. Gastric Cancer. 2015;18(1):65-76. doi:10.1007/s10120-014-0348-0 (Epub 2014/02/11).

7. Maekita T, Nakazawa K, Mihara M, Nakajima T, Yanaoka K, Iguchi M, et al. High levels of aberrant DNA methylation in Helicobacter pylori-infected gastric mucosae and its possible association with gastric cancer risk. Clin Cancer Res. 2006;12(3 Pt 1):989-95. doi:10.1158/1078-0432.ccr-05-2096 (Epub 2006/02/10).

8. Nakajima T, Maekita T, Oda I, Gotoda T, Yamamoto S, Umemura $\mathrm{S}$, et al. Higher methylation levels in gastric mucosae significantly correlate with higher risk of gastric cancers. Cancer Epidemiol Biomark Prev. 2006;15(11):2317-21. doi:10.1158/ 1055-9965.epi-06-0436 (Epub 2006/11/23).

9. Niwa T, Toyoda T, Tsukamoto T, Mori A, Tatematsu M, Ushijima T. Prevention of Helicobacter pylori-induced gastric cancers in gerbils by a DNA demethylating agent. Cancer Prev Res (Philadelphia, PA). 2013;6(4):263-70. doi:10.1158/1940-6207. capr-12-0369 (Epub 2013/04/06).

10. Slaughter DP, Southwick HW, Smejkal W. Field cancerization in oral stratified squamous epithelium; clinical implications of multicentric origin. Cancer. 1953;6(5):963-8 (Epub 1953/09/01).

11. Wang K, Yuen ST, Xu J, Lee SP, Yan HH, Shi ST, et al. Wholegenome sequencing and comprehensive molecular profiling identify new driver mutations in gastric cancer. Nat Genet. 2014;46(6):573-82. doi:10.1038/ng.2983 (Epub 2014/05/13).

12. Ushijima T, Sasako M. Focus on gastric cancer. Cancer Cell. 2004;5(2):121-5 (Epub 2004/03/05).

13. Toyota M, Ahuja N, Suzuki H, Itoh F, Ohe-Toyota M, Imai K, et al. Aberrant methylation in gastric cancer associated with the
CpG island methylator phenotype. Cancer Res. 1999;59(21):5438-42 (Epub 1999/12/20).

14. Kang GH, Lee S, Kim WH, Lee HW, Kim JC, Rhyu MG, et al. Epstein-Barr virus-positive gastric carcinoma demonstrates frequent aberrant methylation of multiple genes and constitutes $\mathrm{CpG}$ island methylator phenotype-positive gastric carcinoma. Am J Pathol. 2002;160(3):787-94. doi:10.1016/s0002-9440(10)649012 (Epub 2002/03/14).

15. Matsusaka K, Kaneda A, Nagae G, Ushiku T, Kikuchi Y, Hino R, et al. Classification of Epstein-Barr virus-positive gastric cancers by definition of DNA methylation epigenotypes. Cancer Res. 2011;71(23):7187-97. doi:10.1158/0008-5472.can-11-1349 (Epub 2011/10/13).

16. Chan AO, Lam SK, Wong BC, Wong WM, Yuen MF, Yeung $\mathrm{YH}$, et al. Promoter methylation of E-cadherin gene in gastric mucosa associated with Helicobacter pylori infection and in gastric cancer. Gut. 2003;52(4):502-6 (Epub 2003/03/13).

17. Kang GH, Lee HJ, Hwang KS, Lee S, Kim JH, Kim JS. Aberrant $\mathrm{CpG}$ island hypermethylation of chronic gastritis, in relation to aging, gender, intestinal metaplasia, and chronic inflammation. Am J Pathol. 2003;163(4):1551-6. doi:10.1016/s00029440(10)63511-0 (Epub 2003/09/26).

18. Miyazaki T, Murayama Y, Shinomura Y, Yamamoto T, Watabe $\mathrm{K}$, Tsutsui $\mathrm{S}$, et al. E-cadherin gene promoter hypermethylation in $H$. pylori-induced enlarged fold gastritis. Helicobacter. 2007;12(5):523-31. doi:10.1111/j.1523-5378.2007.00519.x (Epub 2007/09/01).

19. Perri F, Cotugno R, Piepoli A, Merla A, Quitadamo M, Gentile A, et al. Aberrant DNA methylation in non-neoplastic gastric mucosa of $H$. pylori infected patients and effect of eradication. Am J Gastroenterol. 2007;102(7):1361-71. doi:10.1111/j.15720241.2007.01284.x Epub 2007/05/19).

20. Nakajima T, Enomoto S, Yamashita S, Ando T, Nakanishi Y, Nakazawa K, et al. Persistence of a component of DNA methylation in gastric mucosae after Helicobacter pylori eradication. J Gastroenterol. 2010;45(1):37-44. doi:10.1007/s00535-0090142-7 (Epub 2009/10/13).

21. Chan AO, Peng JZ, Lam SK, Lai KC, Yuen MF, Cheung HK, et al. Eradication of Helicobacter pylori infection reverses E-cadherin promoter hypermethylation. Gut. 2006;55(4):463-8. doi:10.1136/gut.2005.077776 (Epub 2006/01/24).

22. Ushijima T, Nakajima T, Maekita T (2006) DNA methylation as a marker for the past and future. J gastroenterol 41(5):401-407. doi:10.1007/s00535-006-1846-6

23. Nakajima T, Yamashita S, Maekita T, Niwa T, Nakazawa K, Ushijima T. The presence of a methylation fingerprint of Helicobacter pylori infection in human gastric mucosae. Int $\mathrm{J}$ Cancer. 2009;124(4):905-10. doi:10.1002/ijc.24018 (Epub 2008/11/28).

24. Schlesinger Y, Straussman R, Keshet I, Farkash S, Hecht M, Zimmerman J, et al. Polycomb-mediated methylation on Lys27 of histone $\mathrm{H} 3$ pre-marks genes for de novo methylation in cancer. Nat Genet. 2007;39(2):232-6. doi:10.1038/ng1950 (Epub 2007/01/04).

25. Widschwendter M, Fiegl H, Egle D, Mueller-Holzner E, Spizzo $\mathrm{G}$, Marth $\mathrm{C}$, et al. Epigenetic stem cell signature in cancer. Nat Genet. 2007;39(2):157-8. doi:10.1038/ng1941 (Epub 2007/01/ 04).

26. Takeshima H, Yamashita S, Shimazu T, Niwa T, Ushijima T. The presence of RNA polymerase II, active or stalled, predicts epigenetic fate of promoter $\mathrm{CpG}$ islands. Genome Res. 2009;19(11):1974-82. doi:10.1101/gr.093310.109 (Epub 2009/08/05).

27. Takeshima H, Ikegami D, Wakabayashi M, Niwa T, Kim YJ, Ushijima T. Induction of aberrant trimethylation of histone H3 lysine 27 by inflammation in mouse colonic epithelial cells. 
Carcinogenesis. 2012;33(12):2384-90. doi:10.1093/carcin/ bgs294 (Epub 2012/09/15).

28. Kikuyama M, Takeshima H, Kinoshita T, Okochi-Takada E, Wakabayashi M, Akashi-Tanaka S, et al. Development of a novel approach, the epigenome-based outlier approach, to identify tumor-suppressor genes silenced by aberrant DNA methylation. Cancer Lett. 2012;322(2):204-12. doi:10.1016/j.canlet.2012.03. 016 (Epub 2012/03/22).

29. Hur K, Niwa T, Toyoda T, Tsukamoto T, Tatematsu M, Yang $\mathrm{HK}$, et al. Insufficient role of cell proliferation in aberrant DNA methylation induction and involvement of specific types of inflammation. Carcinogenesis. 2011;32(1):35-41. doi:10.1093/ carcin/bgq219 (Epub 2010/10/29).

30. Katsurano M, Niwa T, Yasui Y, Shigematsu Y, Yamashita S, Takeshima H, et al. Early-stage formation of an epigenetic field defect in a mouse colitis model, and non-essential roles of T- and B-cells in DNA methylation induction. Oncogene. 2012;31(3):342-51. doi:10.1038/onc.2011.241 (Epub 2011/06/ 21).

31. Ushijima T. Epigenetic field for cancerization. J Biochem Mol Biol. 2007;40(2):142-50 (Epub 2007/03/31).

32. Ando T, Yoshida T, Enomoto S, Asada K, Tatematsu M, Ichinose $\mathrm{M}$, et al. DNA methylation of microRNA genes in gastric mucosae of gastric cancer patients: its possible involvement in the formation of epigenetic field defect. Int $\mathrm{J}$ Cancer. 2009;124(10):2367-74. doi:10.1002/ijc.24219 (Epub 2009/01/ 24).

33. Nanjo S, Asada K, Yamashita S, Nakajima T, Nakazawa K, Maekita T, et al. Identification of gastric cancer risk markers that are informative in individuals with past $H$. pylori infection. Gastric Cancer. 2012;15(4):382-8. doi:10.1007/s10120-0110126-1 (Epub 2012/01/13).

34. Asada K, Nakajima T, Shimazu T, Yamamichi N, Maekita T, Yokoi C, et al. Demonstration of the usefulness of epigenetic cancer risk prediction by a multicentre prospective cohort study. Gut. 2015;64(3):388-96. doi:10.1136/gutjnl-2014-307094 (Epub 2014/11/08).

35. Asada K, Nakajima T, Shimazu T, Yamamichi N, Maekita T, Yokoi C, et al. Demonstration of the usefulness of epigenetic cancer risk prediction by a multicentre prospective cohort study. Gut. 2014. doi:10.1136/gutjnl-2014-307094.

36. Asaka M, Kato M, Sakamoto N. Roadmap to eliminate gastric cancer with Helicobacter pylori eradication and consecutive surveillance in Japan. J Gastroenterol. 2014;49(1):1-8. doi:10. 1007/s00535-013-0897-8 (Epub 2013/10/29). 